\title{
Phytotherapeutische Praxis bei Magen-Darm-Beschwerden im Kindesalter
}

Ulrike Kastner

Maria Enzersdorf, Österreich

«Bauchweh» ist ein sehr häufig von Kindern geäussertes Symptom, hinter dem sich von der milden Magen-DarmVerstimmung bis hin zum schwerwiegenden Krankheitsbild mit der Notwendigkeit einer chirurgischen Intervention vieles verbergen kann. Im Schul- und Jugendalter kommt noch hinzu, dass die Symptome psychisch überlagert sein können und die exakte Diagnose nicht immer ganz einfach ist.

Bei der Frage nach der richtigen, altersgerechten Therapie kommt das Wissen um die sehr hohe Selbstheilungsrate zum Tragen; viele Befindlichkeitsstörungen sind allein durch diätetische Massnahmen sowie Förderung einer ausgewogenen Ernährung und Bewegung heilbar. Als Spiegel unserer Zeit sind Bauchschmerzen zunehmend durch ungesunde, nicht kindgerechte Ernährung, Bewegungsarmut und ballaststoffarme Kost ausgelöst, abgesehen davon, dass die Inzidenz von Nahrungsmittelunverträglichkeiten, Sensibilitäten oder echten Allergien im Steigen begriffen ist.

Die Phytotherapie bietet vor allem bei den moderaten bis milden Beschwerdebildern, wie z.B. virale Enteritis, Meteorismen, Dyspepsien und funktionelle Verdauungsbeschwerden, ein vielschichtiges Einsatzgebiet. Traditionelle Arzneipflanzen wie die Kamille, die Pfefferminze oder die Malve sind vor allem in der häuslichen Pflege in Verwendung. Zunehmend wird jedoch der Trend zur Einforderung von schneller Abhilfe beobachtet; das Kind soll möglichst bald wieder in Kinderbetreuungseinrichtungen integrierbar sein - Platz und Zeit für Selbstheilung bleiben da kaum noch.

In diesem Zwiespalt unserer Zeit, zum einen die Suche nach dem «Natürlichen» und zum anderen der Wunsch nach möglichst schneller «Wiederherstellung» des «gesunden Kindes», steht nun die Phytotherapie mit all ihren Möglichkeiten und Grenzen, die es gerade bei Kindern zu berücksichtigen gilt. Alarmsymptome wie kritischer Flüssigkeitsverlust, drohende Exsikkose, ungewollter Gewichtsverlust sowie Anzeichen für ein akutes Abdomen dürfen dabei nicht übersehen werden.

Bereits im frühen Säuglingsalter kann Johannisbrotmehl zum Andicken der Milchnahrung (Muttermilch oder For-
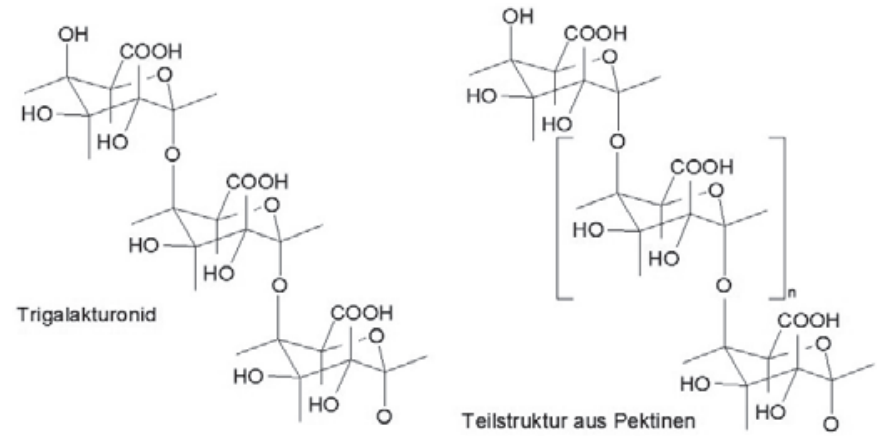

Abb. 1. Struktur der aus der «Karottensuppe» isolierten Trigalakturonide [2].

mula, z.B. Nestargel ${ }^{\circledR}$ ) bei Kindern mit gastroösophagealem Reflux herangezogen werden.

Die typischen sogenannten «Dreimonatskoliken», pathogenetisch nicht immer nur auf schmerzhafte Meteorismen zurückzuführen, sind nach wie vor eine Domäne der Phytotherapie. Zwar haben pharmakologische Studien bezüglich der Toxizität von Bestandteilen des ätherischen Öls als Reinsubstanzen (Estragol) Aufsehen erregt und viel Verunsicherung hervorgerufen, doch kann nach dem derzeitigen Wissensstand davon ausgegangen werden, dass Fencheltee bei bestimmungsgemässem Gebrauch auch im sehr frühen Säuglingsalter bedenkenlos eingesetzt werden kann [1].

Bei der Therapie der infektiösen Enteritis sind traditionelle Konzepte wie getrocknete Heidelbeeren oder Karottensuppe nach Moro hinsichtlich der Wirkprinzipien mittlerweile aufgeklärt. In der Moro'schen Karottensuppe sind es saure Oligosaccharide [2] (Abb. 1), welche die Adhärenz von pathogenen Mikroorganismen an die Darmschleimhaut hemmen und somit einen initialen Schritt in der Pathogenese der Erkrankung unterbinden. Virale und bakterielle Keime werden durch diese Adhärenzblockade rasch und effizient über den Stuhl eliminiert; die Enteritis heilt schneller ab.

Extrakte aus der Heidelbeere finden sich auch als Zusatz zu bilanzierten Glucose-Elektrolyt-Lösungen; die darin enthaltenen Anthocyanidine [3] wirken reizlindernd, entzündungshemmend und adstringierend.

\section{KARGER}

Fax +497614520714

Information@Karger.com

www.karger.com
Dr. med. Ulrike Kastner

Fachärztin für Kinder- und Jugendheilkunde

Hauptstrasse 21, 2344 Maria Enzersdorf, Österreich

ordination@kinderarzt-kastner.at 
Bei Obstipation, die vor allem im Kleinkindesalter zunehmend beobachtet wird, müssen nicht immer synthetische Laxantien oder Klysmen zum Einsatz kommen. Eine konsequente Ernährungsumstellung auf ballaststoffreiche zuckerarme Nahrung sowie der Einsatz von Semen Lini oder Semen Psylli (z.B. Agiolax ${ }^{\circledR}$ mite ohne Senna Granulat, Metamucil ${ }^{\circledR}$ Regular, Mucilar ${ }^{\circledR} /$ Mucilar $^{\circledR}$ avena) können da bereits Abhilfe schaffen. Ist jedoch die Obstipation sehr hartnäckig und wird zu spät diätetisch oder arzneilich interveniert, so ist der Einsatz von Phytotherapeutika meist nicht mehr ausreichend. Anthrachinon-haltige Drogen sind im Kindesalter kontraindiziert.

Bei dyspeptischen Magen-Darm-Beschwerden oder gastritischen Schmerzen ist nach wie vor der klassische Kamillentee, eventuell in Kombination mit anderen antiinflammatorischen und beruhigenden Drogen, indiziert. Auch zugelassene Phytotherapeutika haben sich bei Kindern und Jugendlichen bewährt, so die Kombination eines Presssafts aus der Bitteren Schleifenblume mit Extrakten aus Kümmel, Süssholzwurzel, Pfefferminzblättern, Kamillenblüten, Schöllkraut, Mariendistelfrüchten und Angelikawurzel (Iberogast $\left.{ }^{\circledR}\right)[4]$.
Pfefferminzöl (Colpermin ${ }^{\circledR}$ ) zeigte sich in einer Studie erfolgreich beim kindlichen Reizdarmsyndrom, das sich in der modernen Literatur als eigene Krankheitsentität etabliert hat [5]. Dabei handelt es sich um meist periumbilikale Bauchschmerzen bei 5- bis 15-jährigen Kindern, die unabhängig von Nahrungsmitteln und mit wechselnder Stuhlkonsistenz beobachtet werden. Die Befindlichkeitsstörung ist als Ausschlussdiagnose zu sehen und kann durch immer wiederkehrende Beschwerden und dadurch bedingte häufige Fehlzeiten in der Schule den Alltag eines Kindes und dessen Familie immens beeinträchtigen. In einem Review von Studien an Erwachsenen mit Reizdarmsyndrom hat sich das Pfefferminzöl gegenüber dem Einsatz von synthetischen Spasmolytika als überlegen erwiesen [6].

Für pflanzliche Arzneimittel ist die Vielfalt der Einsatzmöglichkeiten bei Magen-Darm-Erkrankungen im Kindesund Jugendalter auch heute noch gegeben. Sie lassen sich individuell je nach Alter und Beschwerdebild anwenden und bieten eine sichere und kindgerechte Alternative und/oder Ergänzung zu synthetischen Arzneimitteln.

\section{Disclosure Statement}

Keine Interessenkonflikte.

\section{Literatur}

1 Saller R: Keine Angst vor Fencheltee. Schweiz Z Ganzheitsmed 2016;28(suppl 1):10-11.

2 Kastner U, Glasl S, Follrich B, Guggenbichler JP, Jurenitsch J: Saure Oligosaccharide als Wirkprinzip von wäßrigen Zubereitungen aus der Karotte in der Prophylaxe und Therapie von gastrointestinalen Infektionen. Wien Med Wochenschr 2002;152:379-381.
3 Piberger H, Oehme A, Hofmann C, Dreiseitel A, Sand PG, Obermeier F, Schoelmerich J, Schreier P, Krammer G, Rogler G: Bilberries and their anthocyanins ameliorate experimental colitis. Mol Nutr Food Res 2011;55:1724-1729.

4 Ottillinger B, Storr M, Malfertheiner P, Allescher HD: STW 5 (Iberogast ${ }^{\circledR}$ ) - a safe and effective standard in the treatment of functional gastrointestinal disorders. Wien Med Wochenschr 2013;163:65-72.
5 Kline RM, Kline JJ, Di Palma J, Barbero GJ: Enteric-coated, $\mathrm{pH}$-dependent peppermint oil capsules for the treatment of irritable bowel syndrome in children. J Pediatr 2001;138:125-128.

6 Ford AC, Talley NJ, Spiegel BMR, Foxx-Orenstein AE, Schiller L, Quigley EMM, Moayyedi P: Effect of fibre, antispasmodics, and peppermint oil in the treatment of irritable bowel syndrome: systematic review and meta-analysis. BMJ 2008; 337:a2313. 